ReVISTA DE LA ESCUELA de CIENCIAS DE LA EduCACIÓN, AÑo 17, NRO. 16, VOL. 2, JULIO A DiCIEMBRE DE 2021. PÁGINAS 68-77. ISSN 2362-3349 (EN LíNEA). FORMACIÓN DE PROFESIONALES EN INTERACCIÓN CON LA LOCALIDAD EN EL CENTRO UNIVERSITARIO MUNICIPAL DE JAGÜEY GRANDE, CUBA. EDITH ENÉLIDA FARIÑAS BARRIOS. RAQUEL PÉREZ CANO. ADALBERTO PÉREZ SIBILA.

\title{
FORMACIÓN DE PROFESIONALES EN INTERACCIÓN CON LA LOCALIDAD EN EL CENTRO UNIVERSITARIO MUNICIPAL DE JAGÜEY GRANDE, CUBA
}

\author{
Edith Enélida Fariñas Barrios* \\ Universidad Pedagógica Enrique José Varona, Cuba \\ edithfb@ucpejv.edu.cu \\ Raquel Pérez Cano ** \\ Centro Universitario Municipal de Jagüey Grande, Cuba \\ raquel.perez@umcc.cu \\ Adalberto Pérez Sibila** \\ Centro Universitario Municipal de Jagüey Grande, Cuba \\ adalberto.perez@umcc.cu
}

Recibido: 2/06/2020 - Aceptado: 22/11/2020

\section{Resumen}

El vínculo universidad-sociedad demanda la calidad e integración de los procesos y funciones universitarias en correspondencia con la realidad social que garantice el egreso de profesionales integrales, con alta calificación en su desempeño profesional, comprometidos con la transformación, construcción y desarrollo de la sociedad. En los municipios este proceso reclama la relación bidireccional con las entidades locales, pudiendo estas últimas apropiarse de los conocimientos y saberes que aporta la academia en la misma medida que se perfecciona el proceso de formación. En el presente trabajo se presentan reflexiones sobre el comportamiento de la relación CUM-localidad en el proceso de formación de profesionales desde la fundación del Centro Universitario Municipal (CUM) de Jagüey Grande hasta la actualidad. Se presentan acciones dirigidas al perfeccionamiento del proceso de formación en interacción con la localidad y se esbozan algunos resultados enmarcados en tres períodos considerados para el estudio. Se emplearon métodos empíricos de análisis de documentos, encuesta y entrevista que posibilitaron la obtención de resultados sobre la temática de investigación.

Palabras clave: Formación del profesional - Centro Universitario Municipal - Localidad - Interacción.

\footnotetext{
*Licenciada en educación, especialidad Ciencias Biológicas, Máster en Ciencias de la Educación Superior. Actualmente profesora en la Universidad Pedagógica Enrique José Varona en La Habana. Hasta el año 2019 se desempeñó como jefe de departamento en el Centro Universitario Municipal de Jagüey Grande, provincia de Matanzas, Cuba, centro en el que desarrolló investigaciones dirigidas al perfeccionamiento del proceso de formación en pregrado y posgrado en vínculo con la localidad y su integración con los procesos de investigación y extensión universitaria. Los resultados han sido presentados en eventos nacionales e internacionales y publicados en revistas científicas indexadas.

${ }^{*}$ Licenciada en educación en la especialidad Historia y Ciencias Sociales, Máster en Administración de Empresas. Mención Dirección. Actualmente directora del Centro Universitario Municipal de Jagüey Grande, provincia de Matanzas, Cuba, centro en el que desarrolló investigaciones dirigidas a la superación y capacitación en la localidad, gestión del conocimiento y su contribución al desarrollo local, así como la innovación vista desde la gestión de gobierno todas relacionadas con los procesos universitarios y los resultados han sido expuesto en eventos nacionales e internacionales, las últimas publicaciones han sido. ${ }^{* * *}$ Licenciado en educación en la especialidad de Ciencias Bilógicas, profesor Auxiliar. Actualmente Jefe del Departamento Básico del Centro Universitario Municipal de Jagüey Grande, provincia de Matanzas, Cuba. Ha desarrollado actividades investigativas dirigidas a la preparación y capacitación de profesionales del territorio, contribuyendo a la gestión del conocimiento para el desarrollo local y su integración con el gobierno, todas relacionadas con los procesos universitarios. Los resultados han sido presentados en eventos nacionales e internacionales.
} 


\title{
PROFESSIONALS TRAINING IN INTERACTION WITH THE LOCALITY IN THE MUNICIPAL UNIVERSITY CENTER OF JAGÜEY GRANDE, CUBA
}

\begin{abstract}
The professionals training degree process demands the integration of the academic, labor and research components with research and extension in correspondence with the social reality that guarantees the graduation of integral professionals, with high qualification in their professional performance, committed to the transformation, construction and development of society. In municipalities this process facilitates the direct relationship of teachers and students in the context where they reside and work. This paper presents reflections about the behavior of the CUM-locality relationship from its founding at the present the professionals training process at the Municipal University Center in Jagüey Grande and the actions directed to the improvement of the training process in interaction with the locality and some results are outlined. The empirical method of document analysis, survey and interviews made possible to obtain the results on the subject of research.
\end{abstract}

Keywords: Professionals training - Municipal University Center - Locality - Interaction.

\section{Introducción}

Durante más de medio siglo en Cuba se han producido transformaciones socioeconómicas y científico tecnológicas que han demandado cambios a las universidades y fuertes vínculos universidad - sociedad para asegurar el cumplimiento de su misión social, lo que ha devenido una revolución en todos los procesos de la educación superior.

Como continuidad de la política educacional cubana y del proceso de universalización, en el año 2002 la educación superior se extiende a los municipios del país. Es en este momento que se incorporan a las aulas universitarias miles de personas procedentes de diferentes fuentes de ingreso y grupos sociales que hoy integran la pléyade de profesionales que laboran en cada localidad, contribuyendo de este modo a la ampliación de las posibilidades y oportunidades de acceso a la universidad, a multiplicar y extender los conocimientos en aras de favorecer la formación de una cultura general integral de la población, de conquistar la equidad y la justicia social.

Visto de este modo, desde su propia concepción los centros universitarios municipales (CUM), al garantizar la calidad de las funciones y procesos universitarios, se constituyen en agentes importantes en la conquista del desarrollo humano sostenible. Tomando esta premisa como punto de partida y dado el papel central de la educación superior en el desarrollo, estos centros tienen la misión de garantizar oportunidades de acceso a una educación superior de calidad y de "aumentar considerablemente el número de jóvenes y adultos que tienen las competencias necesarias, en particular técnicas y profesionales, para acceder al empleo, el trabajo decente" (ONU/Cepal, 2016, p. 15), transformar su localidad y promover su desarrollo.

Asimismo, representa un desafío para los centros universitarios municipales la preparación y adecuación a las exigencias actuales de sus procesos y funciones, de establecer la integración entre ellos y el vínculo con las instituciones locales, pudiendo determinar sus potencialidades y necesidades e incorporar el conocimiento pue poseen los miembros de la sociedad a la formación de los profesionales. Se trata de "colaborar con variados actores sociales en diversas tareas orientadas a la democratización del conocimiento" (Arocena, 2018, p. 55). El artículo que presentamos contiene ideas sobre el comportamiento del vínculo CUMlocalidad en el proceso de formación de profesionales en el Centro Universitario Municipal de Jagüey Grande, perteneciente a la Universidad de Matanzas, Cuba. Se presentan acciones dirigidas al perfeccionamiento del proceso de formación en interacción con la localidad y se esbozan algunos resultados.

En el desarrollo del trabajo se aplicaron como métodos empíricos: el análisis de documentos que posibilitó el estudio de algunos aspectos y resultados relacionados con el comportamiento del proceso de formación en los diferentes períodos identificados para el estudio; las encuestas a egresados del CUM de diferentes cohortes y las entrevistas a directivos y profesionales de la localidad revelaron las debilidades y los logros existentes en los períodos delimitados entre el 2002 y el 2015, anteriores a la reapertura de carreras en el 2016. Se determinaron y realizaron valoraciones sobre las demandas y potencialidades de las instituciones locales, necesarias a considerar para contribuir a la eficacia del proceso de formación de profesionales en vínculo con la localidad y la calidad del egresado. 
ReVISTA DE LA ESCUELA de CIENCIAS DE LA EdUCACIÓN, AÑo 17, NRO. 16, VOL. 2, JULIO A DiCIEMBRE DE 2021. PÁGINAS 68-77. ISSN 2362-3349 (EN LíNEA). FORMACIÓN DE PROFESIONALES EN INTERACCIÓN CON LA LOCALIDAD EN EL CENTRO UNIVERSITARIO MUNICIPAL DE JAGÜEY GRANDE, CUBA. EDITH ENÉLIDA FARIÑAS BARRIOS. RAQUEL PÉREZ CANO. ADALBERTO PÉREZ SIBILA.

\section{Algunos aspectos teóricos relacionados con la formación de profesionales en integración con el entorno}

Desde el siglo pasado el vínculo universidad - sociedad ha adquirido especial relevancia a partir de considerar la responsabilidad social de la universidad en función de dar respuesta a las demandas sociales y de los impactos resultantes de la calidad de sus procesos y funciones. Lograr "una educación de calidad es la base para mejorar la vida de las personas y el desarrollo sostenible" (ONU/Cepal, 2016, p. 15).

Cuando hablamos de calidad tenemos que ser consecuentes con el contexto y el rol de la educación como proceso que debe adaptarse a su época, de la necesidad de considerar el desarrollo tecnológico y la celeridad en la producción de conocimientos en las diferentes áreas. Desde esta perspectiva se valora la localidad como escenario fundamental para que transcurran de forma eficaz los procesos y funciones universitarias y se garantice la calidad del profesional que egresa, sin perder de vista los acontecimientos que tienen lugar a nivel mundial, regional, nacional, provincial y su incidencia en lo local. En la Declaración de la III Conferencia Regional de Educación Superior en la América Latina y el Caribe (CRES, 2018), se enfatiza que:

La responsabilidad social de la educación superior supone desarrollar compromisos territoriales y transformadores, bajo la perspectiva de una comunidad académica expandida, en la que el egresado es un aliado y transformador social. En ella, el conocimiento, la formación y la acción se imbrican para lograr un desarrollo verdaderamente justo y sostenible (IESALC/UNESCO, 2018, p. 17).

Por ello, en Cuba, el Ministerio de Educación Superior ha trabajado por universalizar la universidad, en garantizar el acceso a los centros de altos estudios y la formación de un profesional integral, caracterizado por un alto compromiso con la transformación del contexto donde se desenvuelve. Los CUM representan un trascendental salto en la materialización y desarrollo de la universalización de la educación superior.

La necesidad de consolidar el compromiso social del CUM con el desarrollo humano y sustentable de su localidad surge con más fuerza en la presente década, como una forma de contribuir a la gestión del conocimiento necesario que le permita adaptarse a los nuevos escenarios y condiciones complejas, con la presencia de un vasto uso de las tecnologías y de redes sociales.

En el 11no. Congreso Internacional de Educación Superior, el Ministro Saborido Loidi (2018) valoró como se han ido implementando en el sector nuevas políticas educacionales y reconoce la necesidad de mantener la equidad y la inclusión en el acceso, así como el incremento de las capacidades de ingreso; enfatiza en la gestión universitaria y la innovación para avanzar en el desarrollo local (Ministro Saborido Loidi, 2018, p. 8).

En este sentido, es necesario destacar el papel que le corresponde a los CUM en la formación de los profesionales que necesita cada localidad para contribuir al desarrollo local y adaptar los saberes a la realidad de su profesión sin estar "al margen de los cambios surgidos ni de las nuevas necesidades. Tiene que estar en relación con su entorno" (Fandos, et. al, 2017, p. 335).

La relación con el entorno es de gran importancia, se trata de trabajar en la búsqueda del conocimiento contextualizado, donde se involucre no solo a las instituciones educativas con la formación de los profesionales, sino también la interrelación entre ellas y el medio donde desarrollan sus procesos, logrando cooperación e intercambio con beneficios para ambos, es decir lograr que la sociedad incorpore los saberes generados por la universidad, se nutra de los profesionales formados y que a su vez la academia incorpore al proceso de formación saberes y experiencias resultantes de la práctica profesional generadas en las instituciones. García Guadilla (2008) afirma lo siguiente:

El conocimiento contextualizado supone alto grado de pertinencia social, donde los que aprenden puedan identificarse y poder así ser actores activos en su propia formación. Un conocimiento pertinente implica una óptima combinación entre los conocimientos abstractos (los universales) y los conocimientos más contextualizados (las culturas locales), relacionados con la comunidad, con las memorias, con la historia, con el entorno en términos generales (García Guadilla, 2008, p. 132).

La producción de nuevos conocimientos a consecuencia del desarrollo de la ciencia y la tecnología, así como los cambios en el contexto socioeconómico nacional e internacional, instan perfeccionar y diseñar nuevas generaciones de planes de estudio en todas las carreras. En la concepción de la generación vigente de estos (planes E), se enfatiza la necesidad de reforzar el vínculo universidad-sociedad desde el propio currículo. De ahí que resulta trascendental tener presente la relación entre necesidades educativas y demandas sociales, las características de los participantes en el proceso educativo y el carácter académico de la educación superior con los diseños y prácticas educativas, de investigación y extensión integradas con la formación.

Se trata de lograr la colaboración entre actores internos y externos donde cada uno aporte sus conocimientos y se generen nuevos aprendizajes individuales y colectivos. La participación de los estudiantes en actividades de extensión les ofrece la posibilidad de desarrollar los modos de actuación de la profesión, al implicarlos en la resolución de problemas profesionales. El intercambio con diferentes sectores sociales, sobre todo con los más vulnerables, fomenta el desarrollo de valores y sentimientos, los responsabiliza y los 
ReVISTA de la Escuela de CIENCIAS DE LA EduCACIÓN, AÑo 17, NRO. 16, VOL. 2, JULIO A DiCIEMBRE DE 2021. PÁGINAS 68-77. ISSN 2362-3349 (EN LíNEA). FORMACIÓN DE PROFESIONALES EN INTERACCIÓN CON LA LOCALIDAD EN EL CENTRO UNIVERSITARIO MUNICIPAL DE JAGÜEY GRANDE, CUBA. EDITH ENÉLIDA FARIÑAS BARRIOS. RAQUEL PÉREZ CANO. ADALBERTO PÉREZ SIBILA.

compromete con la transformación de la sociedad. "La curricularización de la extensión -vale decir, su incorporación a todos los planes de estudio universitarios-aparece, así como clave para mejorar la enseñanza" (Arocena, 2018, p. 62).

Igualmente, es esencial fortalecer la investigación dirigida a transformar el contexto local desde el proceso de formación en pregrado, estableciendo el nexo con los diferentes actores sociales en aras del desarrollo sostenible del municipio, donde el diagnóstico de los problemas, sus manifestaciones y causas constituyan el punto de partida para la elaboración de estrategias de trabajo, propuestas de solución y se establezcan las vías de retroalimentación y evaluación de los cambios generados.

\section{Panorámica del CUM de Jagüey Grande y su función en la formación de profesionales hasta el $2015-2016$}

EI CUM de Jagüey Grande, recientemente, el 5 de octubre de 2019 cumplió 17 años de fundado. Desde su creación se consideran tres períodos para describir el proceso de formación de profesionales en pregrado. Por sus particularidades hasta el 2015-2016 se pueden reconocer dos períodos.

El primer período se podría enmarcar desde su fundación (2002) hasta el curso escolar 2009-2010 caracterizado por el comienzo en la formación de profesionales, acelerado incremento en el acceso y el desarrollo de un intenso trabajo encaminado a la formación pedagógica, metodológica y didáctica de los profesionales que iniciaban su labor docente, tanto desde la superación posgraduada como a través del trabajo metodológico y la investigación. La institución se identificaba como Sede Universitaria Municipal.

En el 2002 solo se cursaban carreras de Humanidades (Estudios Socioculturales, Derecho, Comunicación Social y Psicología). La matrícula inicial fue de 20 estudiantes que accedieron a la modalidad semipresencial, al tipo de curso continuidad de estudios ${ }^{1}$. Estos jóvenes procedían de programas sociales creados con el propósito de elevar la calidad de vida de la población cubana. Posteriormente en el 2003 se incorporaron las carreras Ingeniería Industrial, Contabilidad y Finanzas. Se alcanzó una matrícula total de 120 estudiantes. En el 2008-2009 inicia Turismo.

La constitución del CUM representó un cambio significativo y un impacto positivo para los pobladores. Desde el curso escolar 2005-2006 no solo jóvenes, sino personas de diferentes edades que por diversas razones no tuvieron la posibilidad de acceder a las universidades y mostraron su deseo de cursar estudios superiores pudieron materializar sus aspiraciones y matricularon en los cursos de Educación a Distancia ${ }^{2}$ a nivel municipal, en las carreras Estudios Socioculturales, Derecho y Contabilidad y Finanzas. Esto trajo consigo un incremento en la matrícula, que mostró su mayor cifra en el 2008-2009 con un total de 347 estudiantes en continuidad de estudios y 870 en educación a distancia.

Los logros más relevantes de la interrelación CUM-localidad, como parte del vínculo universidadsociedad, estuvieron dados en que las instituciones locales se convirtieron en escenarios educativos para el desarrollo del proceso docente educativo y en el alto número de profesionales que laboraban en estas instituciones dispuestos a desempeñarse como profesores en las diferentes especialidades, lo que permitió seleccionar aquellos con reconocido prestigio y óptimos resultados en la superación y en las actividades laborales, cualidades esenciales para desarrollar la docencia con calidad.

Una investigación relevante en esta etapa lo constituyó la investigación en la que se diseñó el Modelo teórico metodológico para integrar la formación del profesional a la producción y aplicación de conocimientos, en el que se exponen los fundamentos, que han servido de guía para la toma de decisiones relacionadas con el vínculo CUM - localidad. Se sugirieron acciones, formas y vías de su concreción. Como parte de este estudio se identificaron las instituciones locales productoras de conocimiento (Rodríguez \& Ginoris, 2014). Este Modelo comienza a implementarse desde el curso escolar 2009-2010.

El proceso de formación en interacción con la localidad implica la integración de los procesos y funciones sustantivas de la universidad. En este sentido fue relevante la labor de la carrera Estudios Socioculturales desde los inicios en la creación de variados espacios que promovieron el desarrollo de

\footnotetext{
1 Tipo de curso diseñado específicamente para las sedes universitarias municipales con el propósito de llevar la universidad a todos los municipios de país, ampliar el acceso y dar respuesta a las demandas de superación de los jóvenes beneficiados con los programas sociales. Caracterizado por el desarrollo de actividades presenciales con la utilización de diferentes formas organizativas: clase encuentro, consultas y tutorías complementadas con la autopreparación del estudiante. En el 2011 deja de cumplir su rol y se asume la clasificación normada para la modalidad semipresencial en la educación superior cubana: curso por encuentros.

${ }^{2}$ Modalidad de estudio que amplía las posibilidades de acceso a personas que cuentan con título de nivel medio superior (único requisito para ingresar). Caracterizada por la flexibilidad en la selección de las asignaturas a matricular y cursar en cada período académico, sin afectar la lógica de los contenidos y la interrelación entre ellos. La forma organizativa presencial en esta modalidad es la consulta (colectiva o individual) ofrecida por el profesor con el objetivo de aclarar las dudas que le surgen al estudiante durante la autoprepación y la solución de las guías de estudio. Desde el 2005 hasta el 2011, no se establecían límites en las veces que los estudiantes podían matricular y examinar cada asignatura incluida en el plan de estudio ni en el tiempo para culminar la carrera, avanzaba a su propio ritmo. Posteriormente se limita a cuatro veces las opciones para matricular cada asignatura, respetando la lógica del plan de estudio y se limita a 10 años el tiempo para culminar estudios.
} 
ReVISTA DE LA ESCUELA de CIENCIAS DE LA EdUCACIÓN, AÑo 17, NRO. 16, VOL. 2, JULIO A DiCIEMBRE DE 2021. PÁGINAS 68-77. ISSN 2362-3349 (EN LíNEA). FORMACIÓN DE PROFESIONALES EN INTERACCIÓN CON LA LOCALIDAD EN EL CENTRO UNIVERSITARIO MUNICIPAL DE JAGÜEY GRANDE, CUBA. EDITH ENÉLIDA FARIÑAS BARRIOS. RAQUEL PÉREZ CANO. ADALBERTO PÉREZ SIBILA.

actividades de extensión universitaria a nivel comunitario e involucraron estudiantes en la solución de problemas de la especialidad en beneficio de los pobladores y de su calidad de vida, pudiendo desarrollar la cultura de su profesión, general y comunicacional.

Logro significativo estuvo dado por la atención sistemática que brindaron los directivos y profesionales de las instituciones locales a los estudiantes, procedentes de cursos de superación integral para jóvenes ${ }^{3}$ y Tarea Álvaro Reynoso ${ }^{4}$ vinculados a la actividad de estudio sin otro vínculo laboral, durante la realización de su práctica laboral. En el 2006-2007 se graduaron los tres primeros universitarios, dos Licenciados en Psicología y uno en Derecho. Hasta el 2010 se completaron 414 graduados de las diferentes carreras.

El mayor obstáculo a que nos enfrentamos en los tres primeros años estuvo dado por las condiciones del local donde radicaba la dirección de la institución, espacio pequeño que limitaba el cumplimiento de las actividades colectivas del claustro de profesores, sin embargo, se contó con el apoyo de otras instituciones que brindaron sus instalaciones.

El 96\% del claustro de profesores estaba conformado por profesores a tiempo parcial, es decir, profesionales que laboraban en otras instituciones y alternaban su empleo con la docencia, por lo que fue necesario conciliar con cada uno de ellos y con sus dirigentes los posibles momentos para desarrollar las actividades metodológicas y de superación colectivas. Se dedicó el mayor tiempo a su formación pedagógica, de ahí que predominaron las investigaciones en esta área. No se potenció la actividad científica en relación con la localidad y sus potencialidades para el desarrollo del proceso de formación.

La actividad científica estudiantil fue incipiente en función del desarrollo local; se caracterizó fundamentalmente por la elaboración de trabajos de curso y otras investigaciones extracurriculares, según temas de interés de los estudiantes, profesores o tutores, de los que se presentaron a eventos científicos estudiantiles 137 resultados.

En el 2008-2009 se defienden los primeros trabajos de diploma, inicialmente en número limitado (solo dos). En el 2010-2011 se totalizaron 17. De la misma forma, se presentaron dificultades para integrar las actividades de extensión al proceso de formación, como parte de los programas de las asignaturas en las diferentes carreras.

En los años comprendidos en el período 2010-2011 al 2015- 2016, se identificó el centro como Filial Universitaria Municipal del MES, ya formaba parte del CUM, en este momento como Consejo Universitario Municipal, se daban pasos para la integración de las cuatro filiales existentes en la localidad, pero sin institucionalizar aún. Es a partir del 2013 como parte de la integración de la Universidad cubana que ocurre la unificación, dejan de existir las filiales aisladas, excepto la Filial de Ciencias Médicas y se constituye una única institución, el CUM Jagüey Grande (Centro Universitario Municipal). En este año se incorporan los profesores y estudiantes de Cultura Física y en el 2014 los profesores de carreras pedagógicas, no contaban con estudiantes en las carreras por limitación en el acceso de estudiantes.

Durante estos años se establecen estrategias encaminadas al fortalecimiento de la formación del profesional en los distintos tipos de curso, el perfeccionamiento en la formación continua del claustro, la búsqueda y ampliación de las relaciones con las instituciones productoras de conocimiento existentes en la localidad.

Especial atención recibió la actividad científica estudiantil, se desarrollaron investigaciones dirigidas a dar respuestas a problemas locales. Fueron presentados a eventos 255 trabajos investigativos y de los 611 graduados en estos años, 152 (24,87\%) realizaron trabajo de diploma como ejercicio de culminación de estudios. Se destacan las carreras: Contabilidad y Finanzas (51), Agronomía (48) y Turismo (41).

La extensión universitaria alcanzó relevancia y se desarrollaron variadas actividades, en la mayoría, espacios protagonizados por estudiantes bajo la dirección del profesor e integradas al proceso de formación, sin embargo, la disminución de la matrícula representó un obstáculo para el cumplimiento de los procesos y funciones sustantivas de la universidad y para la satisfacción de las demandas locales.

Representó un momento de discontinuidad en el acceso, no se logró incorporar estudiantes de nuevo ingreso porque no cumplían con el requisito establecido a partir de este momento, referente a examinar y aprobar exámenes de ingreso en las asignaturas: Matemática, Español e Historia de Cuba y es significativo considerar que al CUM accedían y acceden personas que en muchos casos han estado desvinculados de la actividad de estudio. Por esta razón el proceso de formación estuvo dirigido a estudiantes de los diferentes tipos de curso que ingresaron antes de hacerse vigente la normativa.

\footnotetext{
${ }^{3}$ El curso de superación integral para jóvenes fue uno de los programas sociales creados para jóvenes graduados de educación media superior desvinculados del estudio y del trabajo. Se formaron grupos y se les asignaron profesores que durante un año trabajaron en la ejercitación y consolidación de los contenidos vencidos previamente que les pudiera servir de base para apropiarse de los nuevos contenidos al ingresar a la universidad. Se consideró el estudio como empleo con remuneración durante la duración de la carrera. Realizaban prácticas laborales sistemáticas en las instituciones locales con actividades afines a la profesión.

${ }^{4}$ Otro de los programas sociales, dirigido a personas que laboraban en centrales azucareros y fueron cerrados por decisiones estatales en correspondencia con las condiciones económico sociales imperantes en ese momento. Estas personas recibieron un tratamiento similar a los beneficiados con los cursos de superación integral.
} 
ReVISTA de la Escuela de CIENCIAS DE LA EduCACIÓN, AÑo 17, NRO. 16, VOL. 2, JULIO A DiCIEMBRE DE 2021. PÁGINAS 68-77. ISSN 2362-3349 (EN LíNEA). FORMACIÓN DE PROFESIONALES EN INTERACCIÓN CON LA LOCALIDAD EN EL CENTRO UNIVERSITARIO MUNICIPAL DE JAGÜEY GRANDE, CUBA. EDITH ENÉLIDA FARIÑAS BARRIOS. RAQUEL PÉREZ CANO. ADALBERTO PÉREZ SIBILA.

Se realizaron esfuerzos por incorporar estudiantes a las carreras priorizadas para el desarrollo de la localidad, tales como: Agronomía, carreras pedagógicas y Cultura física. Entre las acciones ejecutadas: el intercambio con empleadores con el propósito de establecer estrategias conjuntas para garantizar el ingreso, fundamentalmente con las Delegación de la Agricultura, Direcciones Municipales de Educación y de Deportes.

Se inició un proceso de captación, se formaron grupos con jóvenes aspirantes a estas carreras y se les impartieron las asignaturas Matemática, Español e Historia, pero no fructificaron los esfuerzos y no fue posible la apertura o reapertura de carreras.

\section{Formación de profesionales en el CUM Jagüey Grande desde el 2016 a la actualidad}

Es, a partir del 2016-2017 hasta nuestros días que consideramos el tercer período de existencia de esta institución universitaria local. Se reabrieron carreras en correspondencia con las necesidades de la localidad. Un CUM integrado, con cuatro carreras en Curso por Encuentros (Licenciatura en Educación prescolar, Licenciatura en Educación primaria, Agronomía, Cultura física) y cuatro en Curso a Distancia (Derecho, Contabilidad y finanzas, Gestión sociocultural para el desarrollo y Turismo).

El reinicio y continuidad de los procesos universitarios con la reapertura de las carreras representó un reto para los directivos y el claustro de profesores que implicó a todos en el análisis de las experiencias y resultados de los períodos anteriores; en el estudio de los objetivos de trabajo propuestos por el Ministerio de Educación Superior, de las áreas de resultado clave y de los criterios de medida para ajustar y diseñar los del CUM.

Como respuesta a la política trazada por el Estado, por el Ministerio de Educación Superior y para cumplir con el encargo social otorgado a los CUM de contribuir al desarrollo de las localidades donde están situados, conjuntamente con actores locales se elaboró el Sistema de innovación local encaminado a potenciar la producción de alimentos en la localidad. Uno de sus subsistemas se corresponde con la gestión del proceso de formación para contribuir al desarrollo local.

Toda la información recopilada ha servido de referencia para diseñar, rediseñar y adaptar acciones y actividades encaminadas a establecer la relación bidireccional con las instituciones y organizaciones locales. La elaboración de convenios y proyectos ha permitido establecer el vínculo con entidades laborales reconocidas a nivel nacional y centros docentes para el desarrollo del proceso de formación, entre ellos, Centro de Capacitación de la Empresa Agroindustrial Victoria de Girón, Unidad Científica Tecnológica de Base de Jagüey Grande y Direcciones Municipales de Deportes y Educación. La emisora local Radio Victoria de Girón desde los primeros años ha divulgado resultados del proceso de formación.

Como parte de los convenios y proyectos, se determinó, además la posible propuesta de temas a investigar por los estudiantes, acorde con las necesidades y demandas de cada institución que recibiría el beneficio de los profesionales formados.

El diseño de las acciones se realizó atendiendo a dos direcciones: Dirección 1: Formación continua de los profesores (desde el trabajo metodológico, la investigación y la superación posgraduada). Los resultados relacionados con esta dirección se presentaron previamente por las autoras (Rodríguez et. al., 2015, p.239) (Fariñas y Pérez, 2019, p.92).

Según los objetivos de la investigación referimos las acciones desarrolladas en la Dirección 2 Formación de profesionales en interacción con la localidad (Fariñas et al., 2019, 8,9).

\section{Acciones dirigidas a establecer el vínculo con instituciones y organizaciones de la localidad}

- Determinación de las potencialidades de organizaciones e instituciones de la localidad para desarrollar la formación laboral e investigación en cada carrera, teniendo en cuenta la disciplina, el año y la asignatura. Elaboración de convenios y proyectos, asimismo participación en proyectos de instituciones científicas.

- Análisis y defensa de los planes de estudio de las carreras con la participación de directivos locales, personas que forman parte del potencial científico y profesionales con resultados relevantes en la profesión.

- Preparación a profesionales de la localidad con vasta experiencia preparados para participar en el proceso de formación del profesional.

- Planificación, ejecución y control de actividades para el desarrollo de la actividad laboral investigativa, conjuntamente con profesionales de reconocido prestigio, resultados laborales y científicos que laboran en entidades de la localidad.

- Identificación de problemas existentes en las empresas, instituciones locales y sociales a investigar por los estudiantes.

- Ejecución de proyectos de extensión universitaria, comunitarios y/o de investigación, conjuntamente con instituciones locales.

- Participación de directivos y otros trabajadores de instituciones locales en espacios donde se presentan resultados de investigación de los estudiantes encaminados a la solución de problemas. 
ReVISTA de la Escuela de CIENCIAS DE LA EduCACIÓN, AÑo 17, NRO. 16, VOL. 2, JULIO A DiCIEMBRE DE 2021. PÁGINAS 68-77. ISSN 2362-3349 (EN LíNEA). FORMACIÓN DE PROFESIONALES EN INTERACCIÓN CON LA LOCALIDAD EN EL CENTRO UNIVERSITARIO MUNICIPAL DE JAGÜEY GRANDE, CUBA. EDITH ENÉLIDA FARIÑAS BARRIOS. RAQUEL PÉREZ CANO. ADALBERTO PÉREZ SIBILA.

\section{Acciones dirigidas al desarrollo del proceso docente educativo en interacción con la localidad}

- Aplicación de diagnóstico a estudiantes sobre características personales, de su formación, particularidades para aprender, estrategias, habilidades y procedimientos que constituyen referencia para la atención personalizada.

- Ejecución de actividades en las carreras, las disciplinas y las asignaturas que requieren de la práctica y la ejercitación de habilidades en situaciones organizadas para el aprendizaje y de la consolidación progresiva en la actividad académica, laboral e investigativa.

- Orientación, realización y exposición de resultados de la actividad laboral e investigativa desarrollada.

- Elaboración y solución de ejercicios integradores relacionados con el contexto local en el colectivo de grupo, disciplinas y con enfoque interdisciplinario.

- Incorporación a las actividades curriculares y extracurriculares del trabajo en equipos y del aprendizaje cooperativo en la solución a problemas propios de cada profesión pertinentes al contexto local; en un entorno laboral investigativo.

- Solución de guías de estudio orientadoras y dirigidas a potenciar la independencia cognoscitiva, la autovaloración y autocontrol del estudiante durante la autopreparación.

- La ejecución de las acciones ha devenido un proceso en el que se aprecian avances generales, aunque más perceptibles en determinadas carreras o grupos. Existen evidencias de resultados logrados:

- Se incrementa la respuesta a las demandas de formación de profesionales en el territorio a partir de la reapertura de 6 carreras en el 2016-2017, una en 2018-2019 y otra en 2019-2020. Actualmente con una matrícula de 216 estudiantes en Curso por encuentros y 154 en Curso a Distancia.

- Fortalecimiento de la vinculación entre directivos y potencial científico de las instituciones locales con los profesores del CUM, que incide en la formación integral del profesional.

- Mejora continua de la calidad del proceso docente educativo y de los indicadores promoción limpia y total y permanencia.

- Se incrementan las áreas de empresas y organismos de la localidad utilizados como escenarios educativos que permiten la relación entre lo académico, laboral e investigativo, tales como: áreas de experimentación de la Unidad científico tecnológica de base, fincas de campesinos, organopónicos, áreas deportivas, círculos infantiles y escuelas primarias.

- Mayor relación con los problemas locales relacionados con las diferentes especialidades y propuestas de solución.

- Se desarrollan dos proyectos comunitarios multidisciplinarios con la participación de estudiantes de diferentes carreras. EI CUM es entidad participante en proyectos de investigación, en este caso, en la Unidad científico tecnológica de base que contribuyen al desarrollo de habilidades investigativas de los estudiantes de Agronomía.

- Solución de tareas docentes integradoras, con enfoque intradisciplinario e interdisciplinario, dirigidos a la solución de problemas de la profesión en la localidad. Se materializa en el año académico, con énfasis en la Disciplina Principal Integradora.

- Se ha incrementado la participación en eventos científicos estudiantiles en el curso por encuentros: 72 trabajos presentados por 105 estudiantes, autores y coautores, en el período 2016-2019.

- En los trabajos de curso planificados se integran contenidos de diferentes asignaturas y disciplinas, los que se corresponden con temas solicitados por diferentes entidades.

- En el curso 2019-2020, se graduaron los primeros estudiantes de las carreras pedagógicas Licenciatura en Educación prescolar y en Educación primaria, que han transitado por el plan de estudios E, en vigor desde el 2016-2017. La totalidad de estos profesionales que egresan, desempeñaron durante el proceso de formación, la docencia en instituciones educativas de la localidad, donde desarrollaron las investigaciones que constituyeron sus trabajos de diploma como ejercicio de culminación de estudios. Educadores de estos centros con buen desempeño y óptimos resultados en su labor, en estrecho contacto con profesores de la carrera, aportaron sus experiencias y colaboraron con los estudiantes para el desarrollo de investigaciones como parte de su formación.

Para el diseño e implementación de las acciones, se tuvo en cuenta la información obtenida de los instrumentos aplicados y las vivencias de los profesores en los periodos precedentes.

El análisis de documentos estadísticos del CUM, datos de matrículas y graduados de las diferentes carreras, resultados de los informes semestrales y anuales sobre el proceso de formación de pregrado, de la 
ReVISTA DE LA ESCUELA dE CIENCIAS DE LA EdUCACIÓN, AÑo 17, NRO. 16, VOL. 2, JULIO A DiCIEMBRE DE 2021. PÁGINAS 68-77. ISSN 2362-3349 (EN LíNEA). FORMACIÓN DE PROFESIONALES EN INTERACCIÓN CON LA LOCALIDAD EN EL CENTRO UNIVERSITARIO MUNICIPAL DE JAGÜEY GRANDE, CUBA. EDITH ENÉLIDA FARIÑAS BARRIOS. RAQUEL PÉREZ CANO. ADALBERTO PÉREZ SIBILA.

actividad científica y de extensión universitaria, conservados durante los 17 años de funcionamiento de la institución aportaron la información sobre el vínculo del proceso de formación con la localidad.

\section{Resultados de las encuestas a egresados}

Se encuestaron 57 graduados de diferentes carreras que laboran en diferentes instituciones de la localidad. Muestran insatisfacciones con su proceso de formación en lo referente al desarrollo de destrezas y capacidades para desempeñarse en la profesión, evidenciado en que el 66,6\% evalúa entre uno y tres de una escala de cinco su preparación para adaptarse a diferentes tareas según las diferentes esferas de actuación de su profesión; la totalidad de los encuestados expresa una o dos tareas en las que se siente más capaz para su cumplimiento.

Las capacidades para desarrollar iniciativas ante problemas de la profesión fueron evaluadas entre uno y tres por el $57,89 \%$ y para trabajar en la comunidad por el $54,38 \%$ de los encuestados. El resultado más bajo $(75,4 \%)$ estuvo referido a poseer los conocimientos y habilidades para desarrollar investigaciones a partir de problemas de la profesión. Refieren argumentos tales como: "predominó el estudio de la teoría", "el cumplimiento de la jornada laboral en el centro de trabajo me impedía asistir a las prácticas"," solo investigué para realizar trabajos de curso", "no investigué durante la carrera, solo algunos trabajos en las asignaturas".

Se sienten satisfechos con la preparación recibida para comunicar los resultados de la actividad investigativa profesional y para trabajar en grupos, el 89,47\%.

Al valorar cómo las actividades docentes contribuyeron a su formación en estrecho vínculo con la localidad plantean insatisfacciones en los ítems relacionadas con: la orientación profesional sobre las condiciones reales y actuales en que se desarrollaría su futuro trabajo profesional $(61,4 \%$ ), así como el vínculo con los problemas reales y concretos de la profesión al igual que la relación entre las actividades académicas, laborales e investigativas (75,4\%); los trabajos de curso y diploma realizados no siempre respondieron a problemas de la comunidad (73,68\%). El 85,96\% reconoce que fue insuficiente la tutoría u orientación de los profesionales de organismos e instituciones de la comunidad para su actividad investigativa o vinculación laboral. El ítem con más bajos resultados fue la participación en eventos y jornadas científicas estudiantiles (88.46).

Señalan como mayores deficiencias en su formación el insuficiente vínculo de la teoría con la práctica y sugieren para mejorar el proceso de formación del profesional para su desempeño en la comunidad, el establecer mayor vínculo con empresas, organismos vinculados al perfil de la carrera, atendidos por profesionales de experiencia.

Algunos argumentos ofrecidos por los graduados al responder las encuestas

Egresado de la carrera Psicología: "Tuve profesores con mucha experiencia en la psicología clínica que me expresaban cómo proceder, me orientaron la realización de estudios de caso, pero no pude participar en ninguna sesión de trabajo con ellos para observar cómo lo hacían."

Egresado de la carrera Contabilidad y Finanzas: "Nunca pude realizar actividades prácticas en una empresa, solo solucionar los ejercicios y problemas que me orientaban para el estudio en las diferentes asignaturas."

Egresado de la carrera Contabilidad y Finanzas: "Tuve la suerte de participar en estudios de factibilidad en una empresa importante como parte de mi trabajo de diploma, lo que me permitió ver en la práctica algunas cuestiones relacionadas con mi trabajo actual como contadora, muchas las pude realizar solo al comenzar a trabajar."

Egresado de la carrera de Derecho: "Todos los profesores de la carrera de Derecho tenían experiencia en la asignatura que impartían y nos presentaban ejemplos reales para su análisis, asistí a juicios orales donde pude ver el rol de cada uno, pero no realicé práctica."

Egresado de la carrera Estudios Socioculturales: "Participé en un proyecto y varias actividades de extensión universitaria que me ayudaron a realizar diagnósticos comunitarios, organizar y ejecutar actividades en la comunidad".

De forma general, los graduados que expresan mayores insatisfacciones son los que cursaron estudios en los primeros años del CUM. Sin embargo, aquellos que fueron beneficiados con los programas: cursos de superación integral para jóvenes y Tarea Álvaro Reynoso se sienten satisfechos con las habilidades, destrezas y capacidades desarrolladas, si se tiene en cuenta que ellos durante toda la carrera realizaron sistemáticamente práctica laboral en instituciones locales donde laboraban profesores a tiempo parcial y sus tutores.

A 14 directivos y 6 personas que forman parte del potencial científico de 13 instituciones se les realizaron entrevistas individuales. Con respecto al vínculo con el CUM para la formación de profesionales, los criterios más desfavorables emitidos por los directivos que demuestran debilidades se manifiestan en: no haber sido preparados para participar en el proceso de formación del profesional y para la introducción de resultados del trabajo científico de estudiantes en las instituciones que dirigen.

Reconocen que la relación con el CUM se ha materializado a través de la utilización de locales y otras instalaciones para proceso formativo o eventos científicos, consultas para trabajos investigativos de estudiantes, 
ReVISTA DE LA ESCUELA de CIENCIAS DE LA EdUCACIÓN, AÑo 17, NRO. 16, VOL. 2, JULIO A DiCIEMBRE DE 2021. PÁGINAS 68-77. ISSN 2362-3349 (EN LíNEA). FORMACIÓN DE PROFESIONALES EN INTERACCIÓN CON LA LOCALIDAD EN EL CENTRO UNIVERSITARIO MUNICIPAL DE JAGÜEY GRANDE, CUBA. EDITH ENÉLIDA FARIÑAS BARRIOS. RAQUEL PÉREZ CANO. ADALBERTO PÉREZ SIBILA.

profesionales categorizados que ejercen como profesores a tiempo parcial en pregrado o posgrado y en capacitación a cuadros del territorio.

Con respecto al desempeño profesional de los egresados consideran que están bien preparados y se desempeñan adecuadamente, muestran responsabilidad y disciplina, así como mejoramiento de su desempeño a partir de los estudios realizados, se han desarrollado más socialmente y han elevado su nivel cultural.

\section{Algunos criterios expresados por directivos}

Directivo de empresa agrícola: "En la Empresa laboran ingenieros agrónomos, licenciados en Contabilidad y Finanzas y en Derecho, graduados del CUM. Son responsables, se esfuerzan por aprender; algunos necesitan del apoyo de otros trabajadores para resolver problemas que se les presentan".

"Varios trabajadores de la empresa son profesores a tiempo parcial en el CUM y han apoyado en la preparación de los demás trabajadores, han impartido posgrados con gran aceptación por parte de los participantes". "Locales de la Empresa se utilizan como aulas para estudiantes de la carrera Agronomía".

Directivo de institución cultural: "Dos trabajadores son graduados del CUM, en Comunicación social y Estudios socioculturales. Se expresan muy bien, son creativos, dispuestos realizar actividades en la comunidad y las ejecutan con calidad".

Las personas que forman parte del potencial científico reconocieron que los graduados presentan limitaciones para planificar y ejecutar proyectos de investigación y muestran su disposición para cooperar en su formación de pregrado y posgrado. Han resultado de gran valor los resultados de investigaciones y experiencias aportadas por ellos que sirven de referencia para el estudio y la propuesta de posibles temas a investigar por estudiantes de las diferentes carreras al ser reconocidos como problemas en la localidad.

\section{Conclusiones}

En el presente trabajo se han presentado ideas y resultados sobre el comportamiento del vínculo CUMlocalidad en el proceso de formación de profesionales en el Centro Universitario Municipal de Jagüey Grande, perteneciente a la Universidad de Matanzas, Cuba.

El estudio de documentos aprobados en diferentes eventos internacionales, regionales y nacionales concernientes a la formación de profesionales permite reconocer a los centros universitarios municipales como agentes del desarrollo local, responsables de formar profesionales integrales, dotados de conocimientos, habilidades, valores y modos de actuación para su desempeño en la sociedad.

Los datos e información obtenidos a través del análisis de documentos posibilitaron una valoración inicial de las principales fortalezas y debilidades relacionadas con el proceso de formación en el CUM y su vínculo con la localidad desde su fundación hasta el 2015. Las encuestas y entrevistas aportaron opiniones y puntos de vista sobre la formación de profesionales que corroboraron como debilidades más significativas: la insuficiente o nula realización de la práctica laboral, que les diera la posibilidad de resolver problemas de la profesión en instituciones locales como parte de su formación integral. El exiguo número de investigaciones realizadas por los estudiantes en estos años evidencia limitaciones en la integración del proceso de formación con otros procesos universitarios y su contribución a la formación de los profesionales en y para la localidad.

Sintetizando, las ideas y resultados de investigaciones desarrolladas en el CUM y en otras instituciones municipales en el período 2002 - 2020 demuestran que la formación de profesionales en el centro universitario municipal en interacción con la localidad demanda tener presente aspectos como: condiciones socioeconómicas, científico tecnológicas, potencialidades profesionales y científicas existentes en el territorio para desarrollar con calidad el proceso de formación. Hacer partícipes a las instituciones locales en los análisis del plan de estudio y sus resultados, en la preparación de profesores y profesionales beneficia el desarrollo exitoso del proceso.

En conclusión, buscando la correspondencia entre lo estudiado, lo realizado, los resultados y las condiciones del contexto se procedió, en primer lugar, al estudio local que permitió la identificación de instituciones y profesionales con potencialidades para contribuir al proceso de formación, punto de partida para la proyección de acciones dirigidas a lograr la relación. En segundo lugar, la selección de profesionales de la localidad como profesores y tutores ha contribuido a mejorar los vínculos CUM-localidad. En tercer lugar, el desarrollo de actividades académicas, de extensión e investigaciones en instituciones municipales ha elevado el nivel de satisfacción y la motivación por la actividad profesional y el compromiso con el desarrollo local de los estudiantes.

\section{Referencias bibliográficas}

Arocena, R. (2018). De los principios de la Reforma de Córdoba a la universidad para el desarrollo de América Latina en el siglo https://revistas.unc.edu.ar/index.php/integracionyconocimiento/article/view/20105/20089

Fandos M. R. et. al. (2017). Análisis sobre el aprendizaje y la aplicación de las competencias generales en el contexto laboral Estrategias de colaboración entre la formación profesional, la universidad y la empresa. Educar, 53(2), pp. 333-355. http://dx.doi.org/10.5565/rev/educar.889 
ReVISTA de la Escuela de CIENCIAS DE LA EduCACIÓN, AÑo 17, NRO. 16, VOL. 2, JULIO A DiCIEMBRE DE 2021. PÁGINAS 68-77. ISSN 2362-3349 (EN LíNEA). FORMACIÓN DE PROFESIONALES EN INTERACCIÓN CON LA LOCALIDAD EN EL CENTRO UNIVERSITARIO MUNICIPAL DE JAGÜEY GRANDE, CUBA. EDITH ENÉLIDA FARIÑAS BARRIOS. RAQUEL PÉREZ CANO. ADALBERTO PÉREZ SIBILA.

Fariñas et al. (2019). Formación del profesional de pregrado en el Centro Universitario Municipal de Jagüey Grande. Revista Estudios del Desarrollo Social: Cuba y América Latina. 7(1), 86-99. http://www.revflaso.uh.cu/

Fariñas, E. \& Pérez, R. (2019). El trabajo metodológico en el Centro Universitario Municipal de Jagüey Grande, Cuba: particularidades y experiencias. Revista de la Escuela de Ciencias de la Educación, año 15, 2 (14), 41-50. http://www.revistacseducacion.unr.edu.ar/

García Guadilla, C. (7 de noviembre de 2007). El compromiso social de las universidades. [Conferencia central]. IX Congreso Iberoamericano de Extensión Universitaria Celebración de los 50 años de la Asociación Colombiana de Universidades -Ascun Bogotá, Colombia.

IESALC/UNESCO (11-14 de junio de 2018). [Declaración final]. III Conferencia Regional de Educación Superior en América Latina y el Caribe, Córdoba, Argentina. http://www.cres2018.org/biblioteca/declaracion-final-cres-2018

ONU/CEPAL (2016). Agenda 2030 y los Objetivos de Desarrollo Sostenible Una oportunidad para América Latina y El Caribe. Santiago: publicaciones cepal. http://www.cepal.org/temas/

Rodríguez, T. \& Ginoris, O. (2014). Modelo teórico metodológico para integrar la formación del profesional a la producción y aplicación de conocimientos. Revista Avanzada Científica,17(1), 83-100. http://www.avanzada.idict.cu

Rodríguez et al. (2015). Acciones para la superación del claustro de profesores de la Filial Universitaria Municipal de Jagüey Grande, Cuba. Sus resultados. Revista de la Escuela de Ciencias de la Educación, año 11, número 10, 231-248. http://www.revistacseducacion.unr.edu.ar/

Saborido, J. R. (12-16 de febrero de 2018). La Universidad y la Agenda 2030 de desarrollo sostenible en el centenario de la Reforma Universitaria de Córdoba. Visión desde Cuba. [Conferencia inaugural]. 11no Congreso Internacional de Educación Superior Universidad, Universidad 2018, La Habana, Cuba. 